\title{
Sharp Ratio Inequalities for a Conditionally Symmetric Martingale
}

by

\section{Adam OSĘKOWSKI}

\section{Presented by Stanisław KWAPIEŃ}

Summary. Let $f$ be a conditionally symmetric martingale and let $S(f)$ denote its square function.

(i) For $p, q>0$, we determine the best constants $C_{p, q}$ such that

$$
\sup _{n} \mathbb{E} \frac{\left|f_{n}\right|^{p}}{\left(1+S_{n}^{2}(f)\right)^{q}} \leq C_{p, q}
$$

Furthermore, the inequality extends to the case of Hilbert space valued $f$.

(ii) For $N=1,2, \ldots$ and $q>0$, we determine the best constants $C_{N, q}^{\prime}$ such that

$$
\sup _{n} \mathbb{E} \frac{f_{n}^{2 N-1}}{\left(1+S_{n}^{2}(f)\right)^{q}} \leq C_{N, q}^{\prime}
$$

These bounds are extended to sums of conditionally symmetric variables which are not necessarily integrable. In addition, we show that neither of the inequalities above holds if the conditional symmetry is not assumed.

1. Introduction. The purpose of this paper is to present some new sharp estimates for the square function of a conditionally symmetric martingale. Let us begin with some definitions. Assume $(\Omega, \mathcal{F}, \mathbb{P})$ is a nonatomic probability space, filtered by a nondecreasing family $\left(\mathcal{F}_{n}\right)_{n \geq 0}$ of sub- $\sigma$-fields of $\mathcal{F}$. Let $\mathcal{H}$ be a separable Hilbert space, with norm $|\cdot|$ and scalar product $(\cdot, \cdot)$. Let $f=\left(f_{n}\right)$ be an $\mathcal{H}$-valued martingale, adapted to the filtration $\left(\mathcal{F}_{n}\right)$. The difference sequence $d f=\left(d f_{n}\right)_{n \geq 0}$ of $f$ is given by $d f_{0}=f_{0}$, $d f_{n}=f_{n}-f_{n-1}, n=1,2, \ldots$, and we introduce the square function $S(f)$ of

2010 Mathematics Subject Classification: Primary 60G42; Secondary 60G44.

Key words and phrases: martingale, square function, ratio inequality, self-normalized process. 
the martingale $f$ by

$$
S(f)=\left(\sum_{k=0}^{\infty}\left|d f_{k}\right|^{2}\right)^{1 / 2} .
$$

We will also use the notation $S_{n}(f)=\left(\sum_{k=0}^{n}\left|d f_{k}\right|^{2}\right)^{1 / 2}$ for $n=0,1,2, \ldots$. The inequalities comparing various norms of a martingale and its square function play an important role in both classical and noncommutative probability theory, harmonic analysis and other areas of mathematics. See, for instance, the works by Stein [S1], [S2], Dellacherie and Meyer [DM], Pisier and $\mathrm{Xu}[\mathrm{PX}]$. Let us present some results, closely related to the ones we will study in the paper. In [Bu], Burkholder proved the estimates

$$
\left(p^{*}-1\right)^{-1}\|S(f)\|_{p} \leq\|f\|_{p} \leq\left(p^{*}-1\right)\|S(f)\|_{p}, \quad 1<p<\infty,
$$

where $p^{*}=\max \{p, p /(p-1)\}$. The left inequality is sharp for $1<p \leq 2$ and the right one is sharp for $2 \leq p<\infty$. In the other cases the best constants are not known; however, for $p=1$, the sharp estimate $\|f\|_{1} \leq 2\|S(f)\|_{1}$ was proved by the author in $\mathrm{O}$.

The above inequality generalized the earlier results for dyadic real-valued martingales, due to Khinchin $[\mathrm{K}]$, Littlewood $[\mathrm{L}]$, Paley $[\mathrm{P}]$, Marcinkiewicz [M] and Marcinkiewicz and Zygmund [MZ] (in fact, the concept of a martingale did not appear there, the results concerned the partial sums of Rademacher and Haar series). Recall that a martingale on the Lebesgue unit interval is called dyadic if, for all $n$, its $n$th difference and the norm of the $n+1$-st difference are both constant on the interval $\left[(k-1) / 2^{n}, k / 2^{n}\right)$ for all $k=1, \ldots, 2^{n}$. The problem of determining the optimal constants in (1.1) in the dyadic case was partially solved by Wang in [W]. To be precise, Wang determined the best constants $\mu_{p}, \nu_{p}$ for which the inequalities

$$
\begin{array}{ll}
\|f\|_{p} \leq \mu_{p}\|S(f)\|_{p} & \text { if } 0<p \leq 2 \text { or } p \geq 3, \\
\nu_{p}\|S(f)\|_{p} \leq\|f\|_{p} & \text { if } p \geq 2,
\end{array}
$$

hold for any dyadic martingale $f$ taking values in $\mathcal{H}$. In fact, the inequalities above were studied for a wider class of conditionally symmetric martingales, i.e. those $f$ for which $d f_{n}$ and $-d f_{n}$ have the same distribution conditionally on $\mathcal{F}_{n-1}, n=0,1, \ldots$ (we take $\mathcal{F}_{-1}$ to be a trivial $\sigma$-field).

These results extend the analogous inequalities for a stopped Brownian motion: in [D], Davis studied the optimal constants $\nu_{p}, \mu_{p}$ such that for a standard Wiener process $B=\left(B_{t}\right)$ and any stopping time $\tau$ adapted to $\left(\mathcal{F}_{t}^{B}\right)$,

$$
\begin{array}{ll}
\nu_{p}\left\|\tau^{1 / 2}\right\|_{p} \leq\left\|B_{\tau}\right\|_{p} & \text { if } 1<p<\infty \text { and }\left\|\tau^{1 / 2}\right\|_{p}<\infty, \\
\left\|B_{\tau}\right\|_{p} \leq \mu_{p}\left\|\tau^{1 / 2}\right\|_{p} & \text { if } 0<p<\infty .
\end{array}
$$

Furthermore, the constants $\mu_{p}$ and $\nu_{p}$ above are the same as in 1.2. 
There is a natural question whether other estimates, valid for a stopped Brownian motion, can be extended to the case of Hilbert space valued conditionally symmetric martingales. In this paper we give the affirmative answer in the case of ratio inequalities, considered in the Brownian setting by Pedersen and Peskir $[\mathrm{PP}]$. We prove the following.

THEOREM 1.1. Let $p, q$ be fixed positive numbers. If $p<2 q$, then for any conditionally symmetric martingale $f$,

$$
\sup _{n} \mathbb{E} \frac{\left|f_{n}\right|^{p}}{\left(1+S_{n}^{2}(f)\right)^{q}} \leq z_{*}^{p}\left(M\left(q-\frac{p}{2}, \frac{1}{2}, \frac{z_{*}^{2}}{2}\right)\right)^{-1},
$$

where $M$ is the Kummer hypergeometric function (see Section 2 below), and $z_{*}=z_{*}(p, q)$ is the unique solution to the equation

$$
p M\left(q-\frac{p}{2}, \frac{1}{2}, \frac{z^{2}}{2}\right)=z^{2}(2 q-p) M\left(q-\frac{p}{2}+1, \frac{3}{2}, \frac{z^{2}}{2}\right) .
$$

The constant on the right in (1.3) is the best possible even in the case of real-valued dyadic martingales.

If $p \geq 2 q$, then there is no finite universal $C_{p, q}<\infty$ for which the estimate

$$
\sup _{n} \mathbb{E} \frac{\left|f_{n}\right|^{p}}{\left(1+S_{n}^{2}(f)\right)^{q}} \leq C_{p, q}
$$

holds for any conditionally symmetric martingale $f$.

We also establish the following nonsymmetric ratio inequality for realvalued conditionally symmetric martingales.

TheOrem 1.2. Let $N=1,2, \ldots$ and $q$ be a positive number. If $q>$ $N-1 / 2$, then for any real-valued conditionally symmetric martingale $f$ we have

$$
\sup _{n} \mathbb{E} \frac{f_{n}^{2 N-1}}{\left(1+S_{n}^{2}(f)\right)^{q}} \leq z_{*}^{2 N-1} \exp \left(-z_{*}^{2} / 4\right)\left(D_{2(N-q)-1}\left(-z_{*}\right)\right)^{-1} .
$$

Here $D$ is a parabolic cylinder function (see Section 2) and $z_{*}=z_{*}(N, q)$ is the unique solution to the equation

$$
(2 N-1) D_{2(N-q)-1}(-z)=z(2(q-N)+1) D_{2(N-q-1)}(-z) .
$$

The constant on the right in (1.5) is the best possible even in the case of dyadic martingales.

If $q \leq N-1 / 2$, then there is no universal $C_{N, q}^{\prime}$ for which the inequality

$$
\sup _{n} \mathbb{E} \frac{f_{n}^{2 N-1}}{\left(1+S_{n}^{2}(f)\right)^{q}} \leq C_{N, q}^{\prime}
$$

is valid for any conditionally symmetric martingale $f$. 
In Theorem 1.1, if we replace the conditionally symmetric martingale $f$ and its square function by the stopped Brownian motion $B_{\tau}$ and $\tau$, we get Theorem 2.11 from $[\mathrm{PP}$. Our second result, stated above, generalizes Theorem 2.8 from that paper. There is an interesting question if the inequalities (1.3), 1.5 can be extended (with possibly worse constants) to the case of general martingales. We will prove that the answer is negative: for any parameters $p, q, N$, the ratio inequalities fail to hold in general.

The inequalities (1.3) and (1.5) can be viewed as the optimal bounds for self-normalized martingales. Several results of this type, including moment and tail estimates, can be found in the literature: see e.g. [D1], [D2], [BT]. The advantage of our paper is that we obtain the best constants and study various powers of the martingale in the numerator and the square function in the denominator. There is also another extension of (1.3) and (1.5), which is worth mentioning. As observed in [D2], while studying ratio inequalities it is often possible to relax the integrability assumptions on the processes considered. It will be clear from the proof below that the assertions of Theorems 1.1 and 1.2 still hold if $\left(d f_{n}\right)$ is a sequence of conditionally symmetric variables, possibly nonintegrable.

A few words about the organization of the paper. In the next section we present some basic information on the special functions we need, and describe the results of Pedersen and Peskir. In Section 3, we prove the main inequalites of the paper, while in Section 4 we show the sharpness of these estimates. In the final section we study the ratio inequalities in the case of general martingales.

2. Auxiliary facts and the sketch of the proof. We begin by introducing the special functions which play an important rôle in the paper. For a complete description and the further properties of these objects, the reader is referred to $\mathrm{Ab}$.

The Kummer confluent hypergeometric function is defined by

$$
M(a, b, x)=1+\frac{a}{b} x+\frac{a(a+1)}{b(b+1)} \frac{x^{2}}{2 !}+\ldots
$$

We have

$$
M^{\prime}(a, b, x)=\frac{a}{b} M(a+1, b+1, x) .
$$

Furthermore, if we substitute $\phi(x)=M\left(\nu / 2,1 / 2, x^{2} / 2\right)$, then $\phi$ solves the equation

$$
\phi^{\prime \prime}(x)-x \phi^{\prime}(x)-\nu \phi(x)=0 .
$$


The parabolic cylinder function is defined by the formula

$$
D_{\nu}(x)=A_{1} e^{-x^{2} / 4} M\left(-\frac{\nu}{2}, \frac{1}{2}, \frac{x^{2}}{2}\right)+A_{2} x e^{-x^{2} / 4} M\left(-\frac{\nu}{2}+\frac{1}{2}, \frac{3}{2}, \frac{x^{2}}{2}\right),
$$

where

$$
A_{1}=\frac{2^{\nu / 2}}{\pi^{1 / 2}} \cos (\nu \pi / 2) \Gamma((1+\nu) / 2), \quad A_{2}=\frac{2^{(1+\nu) / 2}}{\pi^{1 / 2}} \sin (\nu \pi / 2) \Gamma(1+\nu / 2) .
$$

The following equality is valid:

$$
\left(e^{x^{2} / 4} D_{\nu}(x)\right)^{\prime}=\nu e^{x^{2} / 4} D_{\nu-1}(x) .
$$

Moreover, the function $\phi(x)=e^{x^{2} / 4} D_{-\nu}(-x)$ is a solution to $(2.2)$. The last property we will need is that for $\nu<0$,

$$
D_{\nu}(x)=\frac{e^{-x^{2} / 4}}{\Gamma(-\nu)} \int_{0}^{\infty} u^{-\nu-1} e^{-x u-u^{2}} d u .
$$

Let us now describe the method of proof of 1.3 and $(1.5)$. In $[\mathrm{PP}$, Pedersen and Peskir studied the optimal stopping problem

$$
V_{*}(t, x)=\sup _{\tau} \mathbb{E}_{x} \frac{\left|B_{\tau}\right|^{p}}{(t+\tau)^{q}}, \quad p, q>0,
$$

where $B$ is a Brownian motion starting from $x$ (under $\mathbb{P}_{x}$ ) and $\tau$ is a stopping time with respect to $\left(\mathcal{F}_{t}^{B}\right)$. They showed that the value function $V_{*}$ is infinite if $q \leq p / 2$, while for $q>p / 2$ it is given by

$$
V_{*}(t, x)= \begin{cases}z_{*}^{p} t^{p / 2-q} M\left(q-\frac{p}{2}, \frac{1}{2}, \frac{x^{2}}{2 t}\right) / M\left(q-\frac{p}{2}, \frac{1}{2}, \frac{z_{*}^{2}}{2}\right) & \text { if }|x| / \sqrt{t}<z_{*}, \\ |x|^{p} / t^{q} & \text { if }|x| / \sqrt{t} \geq z_{*},\end{cases}
$$

where $z_{*}$ is defined in (1.4). This function is the key tool in the proof of (1.3). Let $V:(0, \infty) \times \mathcal{H} \rightarrow \mathbb{R}$ be given by

$$
V(t, x)=z_{*}^{p} t^{p / 2-q} M\left(q-\frac{p}{2}, \frac{1}{2}, \frac{|x|^{2}}{2 t}\right) / M\left(q-\frac{p}{2}, \frac{1}{2}, \frac{z_{*}^{2}}{2}\right) .
$$

Note that the bound on the right of $(1.3)$ equals $V(1,0)$. We will show the following two properties of $V$.

$1^{\circ}$ The majorization property. For any $t>0$ and $x \in \mathcal{H}$ we have

$$
V(t, x) \geq U(t, x):=|x|^{p} / t^{q} .
$$

$2^{\circ}$ The averaging property. For any $t>0$ and $x, d \in \mathcal{H}$,

$$
V\left(t+|d|^{2}, x-d\right)+V\left(t+|d|^{2}, x+d\right) \leq 2 V(t, x) .
$$

Once we establish these two facts, the estimate (1.3) follows. To see this, take a conditionally symmetric martingale $f$ and observe that, by $1^{\circ}$,

$$
\mathbb{E} \frac{\left|f_{n}\right|^{p}}{\left(1+S_{n}^{2}(f)\right)^{q}} \leq \mathbb{E} V\left(1+S_{n}^{2}(f), f_{n}\right) .
$$


Now the averaging property implies that the sequence $\left(V\left(1+S_{k}^{2}(f), f_{k}\right)\right)_{k}$ is a supermartingale. Indeed, by the conditional symmetry of $f$, if $k \geq 1$,

$$
\begin{aligned}
\mathbb{E}\left(V\left(1+S_{k}^{2}(f), f_{k}\right) \mid \mathcal{F}_{k-1}\right)= & \frac{1}{2} \mathbb{E}\left[V\left(1+S_{k-1}^{2}(f)+\left|d f_{k}\right|^{2}, f_{k-1}-d f_{k}\right)\right. \\
& \left.+V\left(1+S_{k-1}^{2}(f)+\left|d f_{k}\right|^{2}, f_{k-1}+d f_{k}\right) \mid \mathcal{F}_{k-1}\right] \\
\leq & V\left(1+S_{k-1}^{2}(f), f_{k-1}\right) .
\end{aligned}
$$

Consequently,

$$
\mathbb{E} \frac{\left|f_{n}\right|^{p}}{\left(1+S_{n}^{2}(f)\right)^{q}} \leq V(1,0),
$$

which is 1.3 . Observe that the argument is still valid if $\left(d f_{n}\right)$ is just a sequence of conditionally symmetric variables.

The proof of 1.5 goes along the same lines. First we look at the optimal stopping problem

$$
W_{*}(t, x)=\sup _{\tau} \mathbb{E}_{x} \frac{B_{\tau}^{2 N-1}}{(t+\tau)^{q}}, \quad N=1,2, \ldots, q>0,
$$

where $B$ and $\tau$ are as previously. Pedersen and Peskir showed that $W_{*} \equiv \infty$ if $q \leq N-1 / 2$, and

$$
W_{*}(t, x)=\left\{\begin{array}{cc}
z_{*}^{2 N-1} t^{N-q-1 / 2} e^{x^{2} / 4 t-z_{*}^{2} / 4} & \\
\times D_{2(N-q)-1}(-x / \sqrt{t}) / D_{2(N-q)-1}\left(-z_{*}\right) & \text { if } x / \sqrt{t}<z_{*}, \\
x^{2 N-1} / t^{q} & \text { if } x / \sqrt{t} \geq z_{*},
\end{array}\right.
$$

if $q>N-1 / 2$. Here $z_{*}$ is given by (1.6). We define

$$
W(t, x)=z_{*}^{2 N-1} t^{N-q-1 / 2} e^{x^{2} / 4 t-z_{*}^{2} / 4} D_{2(N-q)-1}(-x / \sqrt{t}) / D_{2(N-q)-1}\left(-z_{*}\right),
$$

and prove the averaging and the majorization property (with respect to $\left.U(t, x)=x^{2 N-1} / t^{q}\right)$, which yield the desired estimate (1.5).

REMARK 2.1. There is a natural question why we introduce the functions $V, W$ and do not check the majorization and the averaging property of the functions $V_{*}, W_{*}$ instead. The answer is that the calculations for $V_{*}$ and $W_{*}$ are considerably more complex. Fortunately, $V$ and $W$ are sufficient for our purposes.

3. The proofs of the majorization and the averaging property. For $d \in \mathcal{H}$, let $d^{\prime}=d /|d|$ if $d \neq 0$, and $d^{\prime}=0$ otherwise. We will need the following auxiliary fact.

Lemma 3.1. Assume $\psi: \mathbb{R} \rightarrow \mathbb{R}$ is even, of class $C^{2}$ and with the further property that $\psi$ and $\psi^{\prime \prime}$ are convex. Then for any $u, v \in \mathcal{H}$,

$$
\left|\psi^{\prime}(|u|) u^{\prime}-\psi^{\prime}(|v|) v^{\prime}\right| \leq \frac{|u-v|}{2}\left[\psi^{\prime \prime}(|u|)+\psi^{\prime \prime}(|v|)\right] .
$$


Proof. Squaring both sides, we see that the inequality above is equivalent to $A \leq B\left(u^{\prime}, v^{\prime}\right)$, where $A, B$ depend only on $|u|$ and $|v|$. Therefore we only need to check the inequality for $u, v$ satisfying $\left(u^{\prime}, v^{\prime}\right)= \pm 1$. If $\left(u^{\prime}, v^{\prime}\right)=1$, then (3.1) takes form

$$
\left|\psi^{\prime}(|u|)-\psi^{\prime}(|v|)\right| \leq|u-v|\left[\frac{\psi^{\prime \prime}(|u|)+\psi^{\prime \prime}(|v|)}{2}\right],
$$

and follows from

$$
\left|\psi^{\prime}(|u|)-\psi^{\prime}(|v|)\right|=\left|\int_{|v|}^{|u|} \psi^{\prime \prime}(s) d s\right| \leq|| u|-| v|| \cdot \frac{\psi^{\prime \prime}(|u|)+\psi^{\prime \prime}(|v|)}{2} .
$$

Here the latter inequality is a consequence of the convexity of $\psi^{\prime \prime}$. If $\left(u^{\prime}, v^{\prime}\right)=$ -1 , then, since $\psi^{\prime}$ is odd,

$$
\begin{aligned}
\left|\psi^{\prime}(|u|) u^{\prime}-\psi^{\prime}(|v|) v^{\prime}\right| & =\left|\psi^{\prime}(|u|)+\psi^{\prime}(|v|)\right| \\
& =\left|\psi^{\prime}(|u|)-\psi^{\prime}(-|v|)\right|=\left|\int_{-|v|}^{|u|} \psi^{\prime \prime}(s) d s\right| \\
& \leq|| u|+| v|| \cdot \frac{\psi^{\prime \prime}(|u|)+\psi^{\prime \prime}(|v|)}{2} \\
& =|u-v| \cdot \frac{\psi^{\prime \prime}(|u|)+\psi^{\prime \prime}(|v|)}{2} .
\end{aligned}
$$

The proof is complete.

3.1. The proof of the majorization property of the function $V$. This is straightforward. Denote $a=|x| / \sqrt{t}$ and observe the inequality (2.5) is equivalent to

$$
F(a):=\frac{M\left(q-\frac{p}{2}, \frac{1}{2}, \frac{a^{2}}{2}\right)}{a^{p}} \geq \frac{M\left(q-\frac{p}{2}, \frac{1}{2}, \frac{z_{*}^{2}}{2}\right)}{z_{*}^{p}} .
$$

We have, by (2.1),

$$
F^{\prime}(a)=a^{-p-1}\left[a^{2}(2 q-p) M\left(q-\frac{p}{2}+1, \frac{3}{2}, \frac{a^{2}}{2}\right)-p M\left(q-\frac{p}{2}, \frac{1}{2}, \frac{a^{2}}{2}\right)\right],
$$

so $F^{\prime}(a)<0$ for $a<z_{*}$ and $F^{\prime}(a)>0$ for $a>z_{*}$. Thus $F(a) \geq F\left(z_{*}\right)$, which is the claim.

3.2. Proof of the averaging property for the function $V$. Let $\psi: \mathbb{R} \rightarrow \mathbb{R}$ be defined by $\psi(s)=M\left(q-p / 2,1 / 2, s^{2} / 2\right)$. Fix $t>0, x, d \in \mathcal{H}$ and introduce $G=G_{t, x, d}:[0, \infty) \rightarrow \mathbb{R}$ by the formula

$$
G(s)=\left(t+|d|^{2} s^{2}\right)^{p / 2-q}\left[\psi\left(\frac{|x-d s|}{\sqrt{t+|d|^{2} s^{2}}}\right)+\psi\left(\frac{|x+d s|}{\sqrt{t+|d|^{2} s^{2}}}\right)\right] .
$$


As $(2.6)$ is equivalent to $G(1) \leq G(0)$, we will be done if we show that $G$ is nonincreasing. Clearly, we may assume that $d \neq 0$. Denote

$$
s_{1}=\frac{x-d s}{\sqrt{t+|d|^{2} s^{2}}}, \quad s_{2}=\frac{x+d s}{\sqrt{t+|d|^{2} s^{2}}}
$$

and observe that

$$
\begin{aligned}
\frac{G^{\prime}(s)}{\left(t+|d|^{2} s^{2}\right)^{p / 2-q-1}}= & (p-2 q)|d|^{2} s\left[\psi\left(\left|s_{1}\right|\right)+\psi\left(\left|s_{2}\right|\right)\right] \\
& +\psi^{\prime}\left(\left|s_{1}\right|\right)\left[-\left((x-d s)^{\prime}, d\right) \sqrt{t+|d|^{2} s^{2}}-\left|s_{1}\right||d|^{2} s\right] \\
& +\psi^{\prime}\left(\left|s_{2}\right|\right)\left[\left((x+d s)^{\prime}, d\right) \sqrt{t+|d|^{2} s^{2}}-\left|s_{2}\right||d|^{2} s\right] .
\end{aligned}
$$

Now divide through by $\sqrt{t+|d|^{2} s^{2}}|d|$ and note that

$$
\frac{|d| s}{\sqrt{t+|d|^{2} s^{2}}}=\frac{\left|s_{1}-s_{2}\right|}{2}, \quad(x-d s)^{\prime}=s_{1}^{\prime} \quad \text { and } \quad(x+d s)^{\prime}=s_{2}^{\prime} .
$$

Furthermore, as $(p-2 q) \psi(z)=-\psi^{\prime \prime}(z)+z \psi^{\prime}(z)$ for all $z$ (see Section 2), the above equality transforms into

$$
\begin{aligned}
\frac{G^{\prime}(s)}{\left(t+|d|^{2} s^{2}\right)^{p / 2-q-1 / 2}|d|}= & -\frac{\left|s_{1}-s_{2}\right|}{2}\left[\psi^{\prime \prime}\left(\left|s_{1}\right|\right)+\psi^{\prime \prime}\left(\left|s_{2}\right|\right)\right] \\
& -\left(\psi^{\prime}\left(\left|s_{1}\right|\right) s_{1}^{\prime}-\psi^{\prime}\left(\left|s_{2}\right|\right) s_{2}^{\prime}, d^{\prime}\right) .
\end{aligned}
$$

The right hand side does not exceed

$$
-\frac{\left|s_{1}-s_{2}\right|}{2}\left[\psi^{\prime \prime}\left(\left|s_{1}\right|\right)+\psi^{\prime \prime}\left(\left|s_{2}\right|\right)\right]+\left|\psi^{\prime}\left(\left|s_{1}\right|\right) s_{1}^{\prime}-\psi^{\prime}\left(\left|s_{2}\right|\right) s_{2}^{\prime}\right|,
$$

which is nonpositive due to Lemma 3.1 it is straightforward to check that $\psi$ has all the required properties.

3.3. The majorization property for the function $W$. We proceed as previously. Denoting $a=x / \sqrt{t}$ and setting

$$
F(a)=a^{1-2 N} e^{a^{2} / 4} D_{2(N-q)-1}(-a)
$$

we see that we must establish $F(a) \geq F\left(z_{*}\right)$. Using (2.3), we easily check that

$F^{\prime}(a)=-a^{2 N} e^{a^{2} / 4}\left[(2 N-1) D_{2(N-q)-1}(-a)-a(2(q-N)+1) D_{2(N-q)-2}(-a)\right]$, so $F^{\prime}(a)<0$ for $a<z_{*}$ and $F^{\prime}(a)>0$ for $a>z_{*}$. This proves the estimate.

3.4. The averaging property for the function $W$. The arguments are parallel to those used in the proof for the function $V$; however, as we work with processes taking values in $\mathbb{R}$, the calculations are a bit simpler. Let $\varphi(s)=e^{s^{2} / 4} D_{2(N-q)-1}(-s)$ and observe that we must show that for any 
$x \in \mathbb{R}$ and $t>0$, the function $G_{t, x}:[0, \infty) \rightarrow \mathbb{R}$ defined by

$$
G_{t, x}(d)=\left(t+d^{2}\right)^{N-q-1 / 2}\left[\varphi\left(\frac{x-d}{\sqrt{t+d^{2}}}\right)+\varphi\left(\frac{x+d}{\sqrt{t+d^{2}}}\right)\right]
$$

satisfies $G_{t, x}(d) \leq G_{t, x}(0)$ for $d \geq 0$. It is enough to show that $G$ has nonpositive derivative on $(0, \infty)$. Using 2.2 , we see that

$$
G_{t, x}^{\prime}(d)=\left(t+d^{2}\right)^{N-q-1}\left[-\frac{s_{2}-s_{1}}{2}\left(\varphi^{\prime \prime}\left(s_{1}\right)+\varphi^{\prime \prime}\left(s_{2}\right)\right)+\varphi^{\prime}\left(s_{2}\right)-\varphi^{\prime}\left(s_{1}\right)\right],
$$

where $s_{1}<s_{2}$ are given by

$$
s_{1}=\frac{x-d}{\sqrt{t+d^{2}}} \quad \text { and } \quad s_{1}=\frac{x+d}{\sqrt{t+d^{2}}} .
$$

Now observe that $\varphi^{\prime \prime}$ is a convex function: this follows from $(2.3),(2.4)$ and the assumption $q>N-1 / 2$. Therefore

$$
\varphi^{\prime}\left(s_{2}\right)-\varphi^{\prime}\left(s_{1}\right)=\int_{s_{1}}^{s_{2}} \varphi^{\prime \prime}(u) d u \leq \frac{s_{2}-s_{1}}{2}\left(\varphi^{\prime \prime}\left(s_{1}\right)+\varphi^{\prime \prime}\left(s_{2}\right)\right)
$$

and the proof is complete.

4. The sharpness. To prove that the constants appearing in Theorems 1.1 and 1.2 are optimal (in particular, that they are infinite for some parameters $p, q, N)$, one can use the fact that they are the best possible in the case of stopped Brownian motion together with some standard approximation techniques. However, we take here the opportunity to provide a different proof, based on the ideas of Burkholder (see Section 10 in $[\mathrm{Bu}]$ ). We will only deal with the inequality (1.3), as the arguments for (1.5) are essentially the same.

Let $p, q>0$ and suppose that the best upper bound in 1.3 for realvalued dyadic martingales equals $C_{p, q}<\infty$. Arguing as in [Bu], we see that this implies the existence of a function $U:[1, \infty) \times \mathbb{R} \rightarrow \mathbb{R}$ satisfying the following conditions:

(i) $U(1,0) \leq C_{p, q}$,

(ii) $U(t, x) \geq|x|^{p} / t^{q}$ for all $t \geq 1, x \in \mathbb{R}$,

(iii) $U\left(t+d^{2}, x-d\right)+U\left(t+d^{2}, x+d\right)-2 U(t, x) \leq 0$ for all $t \geq 1$, $x, d \in \mathbb{R}$.

Indeed, one takes

$$
U(t, x)=\sup \left\{\mathbb{E}\left|f_{n}\right|^{p} /\left(t-x^{2}+S_{n}^{2}(f)\right)^{q}\right\},
$$

where the supremum is taken over all $n$ and all the simple martingales $f$ starting from $x$ having dyadic differences $d f_{k}, k=1,2, \ldots$.

LEMMA 4.1. The function $U$ is continuous. 
Proof. Observe first that

$$
U(t, x) \leq \liminf _{s \rightarrow t, x^{\prime} \rightarrow x} U\left(s, x^{\prime}\right) .
$$

Indeed, let $f$ be as in the definition of $U(t, x)$. Then by Lebesgue's dominated convergence theorem,

$$
\begin{aligned}
\mathbb{E} \frac{\left|f_{n}\right|^{p}}{\left(t-x^{2}+S_{n}^{2}(f)\right)^{q}} & =\liminf _{s \rightarrow t, x^{\prime} \rightarrow x} \mathbb{E} \frac{\left|x^{\prime}-x+f_{n}\right|^{p}}{\left(s-\left(x^{\prime}\right)^{2}+S_{n}^{2}\left(x^{\prime}-x+f\right)\right)^{q}} \\
& \leq \liminf _{s \rightarrow t, x^{\prime} \rightarrow x} U\left(s, x^{\prime}\right),
\end{aligned}
$$

as $x^{\prime}-x+f$ is simple, starts from $x^{\prime}$ and has dyadic differences except for the first one. Now take the supremum over $f$ to obtain (4.1).

Now, if $x, x^{\prime} \neq 0$, then

$$
U(t, x) \geq\left(\frac{x}{x^{\prime}}\right)^{p-2 q}\left(\max \left\{\frac{t}{s}\left(\frac{x^{\prime}}{x}\right)^{2}, 1\right\}\right)^{-q} U\left(s, x^{\prime}\right) .
$$

To prove this, take $f^{\prime}$ as in the definition of $U\left(s, x^{\prime}\right)$ and let $f=\left(x / x^{\prime}\right) f$. We have

$$
\begin{aligned}
t-x^{2}+S_{n}^{2}(f) & =\left(\frac{x}{x^{\prime}}\right)^{2}\left[t\left(\frac{x^{\prime}}{x}\right)^{2}-\left(x^{\prime}\right)^{2}+S_{n}^{2}\left(f^{\prime}\right)\right] \\
& \leq\left(\frac{x}{x^{\prime}}\right)^{2} \max \left\{\frac{t}{s}\left(\frac{x^{\prime}}{x}\right)^{2}, 1\right\}\left[s-\left(x^{\prime}\right)^{2}+S_{n}^{2}\left(f^{\prime}\right)\right] .
\end{aligned}
$$

Therefore

$$
\begin{aligned}
& \frac{\left|f_{n}\right|^{p}}{\left(t-x^{2}+S_{n}^{2}(f)\right)^{q}} \\
& \quad \geq\left(\frac{x}{x^{\prime}}\right)^{p-2 q}\left(\max \left\{\frac{t}{s}\left(\frac{x^{\prime}}{x}\right)^{2}, 1\right\}\right)^{-q} \frac{\left|f_{n}^{\prime}\right|^{p}}{\left(s-\left(x^{\prime}\right)^{2}+S_{n}^{2}\left(f^{\prime}\right)\right)^{q}} .
\end{aligned}
$$

As $f$ is simple, starts from $x$ and has dyadic differences, the left-hand side can be majorized by $U(t, x)$. Taking the supremum over $f^{\prime}$ yields $(4.2)$. Together with (4.1), this shows that $U$ is continuous on $[1, \infty) \times(\mathbb{R} \backslash\{0\})$.

Finally, since $U(t, x)=U(t,-x)$ (which is an immediate consequence of the definition), the property (iii) and (4.2) imply

$$
\begin{aligned}
U(t, 0) & \geq \frac{U\left(t+x^{2}, x\right)+U\left(t+x^{2},-x\right)}{2}=U\left(t+x^{2}, x\right) \\
& \geq\left(\max \left\{\frac{t+x^{2}}{s}, 1\right\}\right)^{-q} U(s, x) .
\end{aligned}
$$

Combining this with 4.1) yields the claim.

Now extend $U$ to the whole $\mathbb{R}^{2}$ by setting $U(t, x)=U(1, x)$ for $t<1$. Let $\delta>0$ and convolve $U$ with a nonnegative smooth function $g^{\delta}$ satisfying 
$\left\|g^{\delta}\right\|_{1}=1$ and supported on the ball centered at $(0,0)$ and radius $\delta$. As result, we obtain a smooth function $U^{\delta}$ for which (iii) is still valid. Dividing this inequality by $d^{2}$ and letting $d \rightarrow 0$ gives $U_{t}^{\delta}+\frac{1}{2} U_{x x}^{\delta} \leq 0$. Now let $B$ be a standard Brownian motion and $\tau$ be a stopping time for $B$. For $N=1,2, \ldots$, define $\sigma_{N}=\inf \left\{t:\left|B_{t}\right|=N\right\}$. By Itô's formula, for any $N$, $\mathbb{E} U^{\delta}\left(1+\tau \wedge \sigma_{N}, B_{\tau \wedge \sigma_{N}}\right) \leq U^{\delta}(1,0)$. Now let $\delta \rightarrow 0$ and use the continuity of $U$ and Lebesgue's dominated convergence theorem (we have used the stopping times $\sigma_{N}$ in order to guarantee the boundedness) to obtain $\mathbb{E} U(1+$ $\left.\tau \wedge \sigma_{N}, B_{\tau \wedge \sigma_{N}}\right) \leq U(1,0)$. Applying (i) and (ii), we get

$$
\mathbb{E} \frac{\left|B_{\tau \wedge \sigma_{N}}\right|^{p}}{\left(1+\left(\tau \wedge \sigma_{N}\right)\right)^{q}} \leq C_{p, q} .
$$

Now let $N \rightarrow \infty$ and use Fatou's lemma to get

$$
\mathbb{E} \frac{\left|B_{\tau}\right|^{p}}{(1+\tau)^{q}} \leq C_{p, q} .
$$

This implies $C_{p, q} \geq V_{*}(1,0)=V(1,0)$ and completes the proof.

5. Lack of the ratio inequalities in the general case. In this section we show that no ratio inequalities hold for general martingales. We will focus on (1.3) only, the inequality (1.5) can be dealt with exactly in the same manner. Again we will exploit Burkholder's technique. Let $p, q>0$ be fixed and suppose that the best upper bound in 1.3 equals $C_{p, q}<\infty$. Then there exists a function $U$ with the following properties:

(i) $U(1,0) \leq C_{p, q}$,

(ii) $U(t, x) \geq|x|^{p} / t^{q}$ for all $t \geq 1, x \in \mathbb{R}$,

(iii) $\alpha U\left(t+d_{1}^{2}, x+d_{1}\right)+(1-\alpha) U\left(t+d_{2}^{2}, x+d_{2}\right)-U(t, x) \leq 0$ for all $x \in \mathbb{R}$, $t \geq 1, \alpha \in(0,1)$ and $d_{1}, d_{2} \in \mathbb{R}$ satisfying $\alpha d_{1}+(1-\alpha) d_{2}=0$.

Indeed, one takes

$$
U(t, x)=\sup \left\{\mathbb{E}\left|f_{n}\right|^{p} /\left(t-x^{2}+S_{n}^{2}(f)\right)^{q}\right\},
$$

where the supremum is taken over all $n$ and all the simple martingales $f$ starting from $x$.

Fix $x \in \mathbb{R}, t \geq 1, \alpha \in(0,1)$ and $d_{1}>0$. Since $U \geq 0$ (which follows from the very definition), the property (iii) implies $\alpha U\left(t+d_{1}^{2}, x+d_{1}\right) \leq U(t, x)$. Letting $\alpha \rightarrow 1$ we obtain

$$
U\left(t+d_{1}^{2}, x+d_{1}\right) \leq U(t, x),
$$

which, by induction, leads to

$$
U\left(t+N d_{1}^{2}, x+N d_{1}\right) \leq U(t, x)
$$


for any nonnegative integer $N$. Take $x=0, t=1$ and apply (i) and (ii) to get

$$
\frac{\left(N d_{1}\right)^{p}}{\left(1+N d_{1}^{2}\right)^{q}} \leq C_{p, q}
$$

It suffices to take $d_{1}=1 / \sqrt{N}$ and let $N \rightarrow \infty$ to obtain a contradiction. This proves the claim.

Acknowledgments. This research was partially supported by Foundation for Polish Science and MNiSW Grant N N201 397437.

\section{References}

[Ab] M. Abramowitz and I. A. Stegun (eds.), Handbook of Mathematical Functions with Formulas, Graphs and Mathematical Tables, Reprint of the 1972 edition, Dover Publ., New York, 1992.

[BT] B. Bercu and A. Touai, Exponential inequalities for self-normalized martingales with applications, Ann. Appl. Probab. 18 (2008), 1848-1869.

[Bu] D. L. Burkholder, Explorations in martingale theory and its applications, in: École d'Été de Probabilités de Saint-Flour XIX-1989, Lecture Notes in Math. 1464, Springer, Berlin, 1991, 1-66.

[D] B. Davis, On the $L_{p}$ norms of stochastic integrals and other martingales, Duke Math. J. 43 (1976), 697-704.

[D1] V. H. De La Peña, A general class of exponential inequalities for martingales and ratios, Ann. Probab. 27 (1999), 537-564.

[D2] V. H. De La Peña, M. J. Klass and T. L. Lai, Self-normalized processes: Exponential inequalities, moment bounds and iterated logarithm law, ibid. 32 (2004), 1902-1933.

[DM] C. Dellacherie and P. A. Meyer, Probabilities and Potential B, North-Holland, Amsterdam, 1982.

[K] A. Khintchine [A. Ya. Khinchin], Über dyadische Brüche, Math. Z. 18 (1923), 109-116.

[L] J. E. Littlewood, On bounded bilinear forms in an infinite number of variables, Quart. J. Math. Oxford 1 (1930), 164-174.

[M] J. Marcinkiewicz, Quelques théorèmes sur les séries orthogonales, Ann. Soc. Polon. Math. 16 (1937), 84-96.

[MZ] J. Marcinkiewicz et A. Zygmund, Quelques théorèmes sur les fonctions indépendantes, Studia Math. 7 (1938), 104-120.

[O] A. Osękowski, Two inequalities for the first moment of a martingale, its square and maximal function, Bull. Polish Acad. Sci. Math. 53 (2005), 441-449.

[P] R. E. A. C. Paley, A remarkable series of orthogonal functions I, Proc. London Math. Soc. 34 (1932), 241-264.

[PP] J. L. Pedersen and G. Peskir, Solving non-linear optimal stopping problems by the method of time-change, Stochastic Anal. Appl. 18 (2000), 811-835.

[PX] G. Pisier and Q. Xu, Non-commutative martingale inequalities, Comm. Math. Phys. 189 (1997), 667-698.

[S1] E. M. Stein, The development of square functions in the work of A. Zygmund, Bull. Amer. Math. Soc. 7 (1982), 359-376. 
[S2] E. M. Stein, Harmonic Analysis: Real-Variable Methods, Orthogonality, and Oscillatory Integrals, Princeton Univ. Press, Princeton, NJ, 1993.

[W] G. Wang, Sharp square-function inequalities for conditionally symmetric martingales, Trans. Amer. Math. Soc. 328 (1991), 393-419.

Adam Osękowski

Department of Mathematics, Informatics and Mechanics

University of Warsaw

Banacha 2

02-097 Warszawa, Poland

E-mail: ados@mimuw.edu.pl

Received March 25, 2010 